\title{
Disruption of ureide degradation affects plant growth and development during and after transition from vegetative to reproductive stages
}

Hiroshi Takagi ${ }^{1,3^{*}}$ (D), Shunsuke Watanabe ${ }^{1,4}$, Shoma Tanaka', Takakazu Matsuura², Izumi C. Mori ${ }^{2}$, Takashi Hirayama², Hiroshi Shimada ${ }^{1}$ and Atsushi Sakamoto ${ }^{1 *}$

\begin{abstract}
Background: The ureides allantoin and allantoate are major metabolic intermediates of purine catabolism with high nitrogen-to-carbon ratios. Ureides play a key role in nitrogen utilization in ureide-type legumes, but their effects on growth and development in non-legume plants are poorly understood. Here, we examined the effects of knocking out genes encoding ureide-degrading enzymes, allantoinase (ALN) and allantoate amidohydrolase (AAH), on the vegetative-to-reproductive transition and subsequent growth of Arabidopsis plants.

Results: The ureide-degradation mutants (aln and aah) showed symptoms similar to those of nitrogen deficiency: early flowering, reduced size at maturity, and decreased fertility. Consistent with these phenotypes, carbon-tonitrogen ratios and nitrogen-use efficiencies were significantly decreased in ureide-degradation mutants; however, adding nitrogen to irrigation water did not alleviate the reduced growth of these mutants. In addition to nitrogen status, levels of indole-3-acetic acid and gibberellin in five-week-old plants were also affected by the aln mutations. To test the possibility that ureides are remobilized from source to sink organs, we measured ureide levels in various organs. In wild-type plants, allantoate accumulated predominantly in inflorescence stems and siliques; this accumulation was augmented by disruption of its catabolism. Mutants lacking ureide transporters, ureide permeases 1 and 2 (UPS1 and UPS2), exhibited phenotypes similar to those of the ureide-degradation mutants, but had decreased allantoate levels in the reproductive organs. Transcript analysis in wild-type plants suggested that genes involved in allantoate synthesis and ureide transport were coordinately upregulated in senescing leaves.

Conclusions: This study demonstrates that ureide degradation plays an important role in supporting healthy growth and development in non-legume Arabidopsis during and after transition from vegetative to reproductive stages.
\end{abstract}

Keywords: Allantoate, Allantoin, Arabidopsis thaliana, Catabolic pathway, Nitrogen remobilization, Phytohormone, Purine base, Ureide transport

\footnotetext{
* Correspondence: htakagi@umn.edu; ahkkao@hiroshima-u.ac.jp

${ }^{1}$ Graduate School of Science, Hiroshima University, 1-3-1 Kagamiyama,

Higashi-Hiroshima 739-8526, Japan

Full list of author information is available at the end of the article
}

(C) The Author(s). 2018 Open Access This article is distributed under the terms of the Creative Commons Attribution 4.0 International License (http://creativecommons.org/licenses/by/4.0/), which permits unrestricted use, distribution, and reproduction in any medium, provided you give appropriate credit to the original author(s) and the source, provide a link to the Creative Commons license, and indicate if changes were made. The Creative Commons Public Domain Dedication waiver (http://creativecommons.org/publicdomain/zero/1.0/) applies to the data made available in this article, unless otherwise stated. 


\section{Background}

Plants require nitrogen $(\mathrm{N})$ in large quantities and its limited availability negatively impacts plant growth. Therefore, plants recycle and redistribute stored $\mathrm{N}$ in cellular macromolecules (i.e., proteins and nucleic acids) [1, 2]. Although chloroplast proteins, particularly ribulose-1,5-bisphosphate carboxylase/oxygenase, are the main sources of remobilized $\mathrm{N}$ [3-5], nucleic acids are also $\mathrm{N}$ sources [6,7]. Purines and pyrimidines constitute the nitrogenous components of nucleic acids. The free forms of these nucleobases provide reusable $\mathrm{N}$ upon decomposition [8, 9]. Based on $\mathrm{N}$ content, purines are more valuable than pyrimidines, with four ring $\mathrm{N}$ atoms that are recycled eventually as ammonium.

Unless they are converted to nucleotides through salvage reactions, the free forms of purine nucleobases converge on xanthine, the first common intermediate of the purine catabolic pathway. In Arabidopsis [Arabidopsis thaliana (L.) Heynh.], xanthine is further degraded through a series of biochemical reactions that are mediated by seven enzymes distributed in the cytosol, peroxisomes, and endoplasmic reticulum (ER) $[10,11]$. Xanthine is oxidized to urate by xanthine dehydrogenase $(\mathrm{XDH})$ in the cytosol [12, 13]. Although there are two orthologous $\mathrm{XDH}$ proteins (AtXDH1 and AtXDH2), AtXDH1 is mostly responsible for $\mathrm{XDH}$ activities in Arabidopsis $[14,15]$. Urate is then transported into the peroxisomes and converted to the ureide allantoin via three enzymatic reactions. The first reaction is mediated by urate oxidase, followed by the bifunctional enzyme allantoin synthase that catalyzes the last two reactions [16, 17]. Subsequent ureide degradation is accomplished stepwise by four ER-localized hydrolases, releasing four ammonium ions from one molecule of ureide. Allantoin is decyclized by allantoinase (ALN or allantoin amidohydrolase) to allantoate [18], which is degraded by allantoate amidohydrolase (AAH) to ureidoglycine and an ammonium ion [19]. Ureidoglycine is further hydrolyzed to release another ammonium ion by ureidoglycine aminohydrolase [20]. The resultant ureidoglycolate is finally metabolized to hydroxyglycine and then to glyoxylate and two ammonium ions, by ureidoglycolate amidohydrolase and subsequent non-enzymatic decomposition [21]. The purine-derived ammonium is likely re-assimilated for glutamine synthesis in the cytosol, as is that derived from proteolysis and subsequent amino acid deamination, although little is known about how this inorganic ion is exported from the ER. Although the purine-derived ureides allantoin and allantoate are the principal stores of fixed $\mathrm{N}_{2}$ in leguminous species of tropical and sub-tropical origin [22], the precise contribution of $\mathrm{N}$ derived from purines to general nitrogen metabolism remains largely unexplored in non-leguminous plants such as Arabidopsis [10].
Purine-catabolic mutants of Arabidopsis have been isolated, such as those lacking or with reduced expression of $X D H, A L N$, or $A A H$, and thus cannot use purine-derived $\mathrm{N}$ because of the block in ureide synthesis or degradation. A previous study in our laboratory demonstrated that simultaneous post-transcriptional gene silencing (PTGS) of AtXDH1 and AtXDH2 caused growth impairments and early senescence [23]. Similarly, other groups observed that PTGS and a T-DNA insertion mutation of AtXDH1 caused early senescence $[24,25]$. It was proposed that the ureides allantoin and allantoate function as antioxidant agents, and the phenotypes of $x d h$ mutants were attributed to over-oxidation of living cells [24]. On the other hand, the effect of impaired ureide degradation on Arabidopsis growth has not been well described. Earlier studies reported that T-DNA insertion mutants of $A L N$ and $A A H$ (aln and $a a h$ ) did not show any obvious morphological phenotype compared to wild type (WT) plants during early growth on agar medium plates containing sufficient inorganic $\mathrm{N}[18$, $19,26]$. However, $\mathrm{N}$ recycling and remobilization are vigorously activated during and after the transition from vegetative to reproductive stages [2]. Thus, to investigate the physiological importance of ureide-derived $\mathrm{N}$ in Arabidopsis, the effects of defects in ureide degradation should be evaluated at later developmental stages. Our recent studies revealed that allantoin accumulation resulted in increased levels of abscisic acid (ABA) and jasmonic acid (JA) and enhanced stress hormone responses in young Arabidopsis seedlings [27, 28]. It is not known whether altered hormone levels persist and cause long-term effects on the subsequent growth and development of the ureide-degradation mutants.

Members of the ureide permease (UPS) family are regarded as ureide transporters. Certain members of the UPS family, such as PvUPS1 from French bean (Phaseolus vulgaris L.) and GmUPS1-1 and GmUPS12 from soybean (Glycine $\max$ L.), have been shown to mediate symplasmic transport and xylem loading of allantoin in nodulated roots [29-31]. PvUPS1 is possibly also involved in phloem loading of allantoin [32]. Although no evidence has so far been presented for long-distance translocation of the ureides in Arabidopsis, its genome contains five paralogous genes encoding UPS (AtUPS1-5). Among these paralogs, experiments involving heterologous expression in yeast and Xenopus oocytes have demonstrated that AtUPS1, AtUPS2, and AtUPS5 transport allantoin [33-35]. However, AtUPS1 and AtUPS2 exhibited several-fold higher affinity for uracil (AtUPS1 and AtUPS2) over allantoin, suggesting that their primary function in planta is providing the substrate to the uracil salvage pathway rather than transporting ureides [34, 35]. 
In this study, we conducted a detailed evaluation of the growth phenotype of Arabidopsis mutants defective in ureide degradation ( $a l n$ and $a a h$ ). We also conducted experiments to understand the underlying mechanism of growth attenuation of ureide-degrading mutants. Our results show that ureide degradation is important for the growth and development of Arabidopsis during and after the transition from vegetative to reproductive growth under long-day conditions. We also found $\mathrm{N}$ use efficiency (NUE) and endogenous phytohormone levels are affected in ureide-degradation mutants.

\section{Results}

Disruption of ureide degradation accelerates the transition to reproductive growth under long-day conditions

The aln-1 and aln-2 mutants carry loss-of-function alleles of $A L N$ and are incapable of degrading allantoin; the aah mutant is deficient in allantoate hydrolysis due to the disruption of $A A H$. For more detailed information about the aln-1, aln-2 and aah mutants, see Supporting Information Figs S1 and Figure S3 in Watanabe et al. [27]. In T-DNA insertion mutant lines in general, there could be mutations in addition to the T-DNA-tagged mutations of interest [36]. For this reason, we cannot exclude the possibility that mutant phenotypes that are only observed with a single mutant allele are due to inadvertent mutations. Therefore, subsequently we only consider the mutant phenotypes consistent between two independent mutant alleles of the $A L N$ gene as true aln mutant phenotypes. In our laboratory conditions, consistent with previous reports $[18,19,26]$, seedlings of aln-1, aln-2 and aah mutants grown on half-strength Murashige and Skoog (1/2 MS) standard medium containing either gellan gum or agar did not show obvious morphological phenotypes before bolting [27, 28]. Although these mutants produced flowers as reported by Yang and Han [18], we noticed that aah plants bolted earlier than WT plants when plants were grown on $1 / 2$ MS medium plates for 3 weeks (Additional file 1: Figure S1). To further test for early flowering phenotypes, we cultivated $a l n-1$, aln-2 and aah mutants in soil for a longer time. Consistently, aah plants as well as both aln (aln-1 and aln-2) mutants started bolting and flowering as early as 4 weeks after germination (WAG), whereas WT plants remained non-bolting (Fig. 1a), suggesting the early flowering phenotype shown in Additional file 1: Figure S1 is not attributable to the growth conditions. The number of rosette leaves when the primary inflorescence stems emerged was about 14 in WT, but fewer than 10 in ureide-degradation mutants (Fig. 1b). The three mutants also displayed slight but significant decreases in foliar chlorophyll (Additional file 2: Figure
S2). At 5 WAG, although the mutants and WT had similar fresh weights (FW) of the entire aerial parts, the mutants had less FW of rosette leaf blades and more FW of the stems and cauline leaves compared to wild type (Fig. 1c, d). Leaf blades and petioles of the ureide-degradation mutants were significantly shorter than WT (Additional file 3: Table S1). Together, these phenotypes indicated that disruption of ureide degradation accelerated the transition from vegetative to reproductive growth under long-day conditions.

\section{Disruption of ureide degradation impairs reproductive growth}

Since purine catabolism is a putative N-recycling metabolism and early flowering is a typical $\mathrm{N}$-deficient symptom, we hypothesized that ureide degradation substantially contributes to efficient $\mathrm{N}$ use, especially in later growth stages. To test this idea, we examined the aln and aah mutant phenotypes at the late growth stages. At 9 WAG, the three mutants were smaller than the WT, with whole-plant dry weights (DW) approximately half that of WT plants (Fig. 2a). Due to the early-flowering phenotype, the mutants (particularly the aah mutants) tended to form more siliques than did WT plants during the early stages of inflorescence development (up to 7 WAG; Fig. 2b). However, consistent with the lower DW, total silique numbers of the three mutants were reduced to 50 to $67 \%$ of WT at 9 WAG (Fig. 2b). Moreover, the siliques of these mutants were significantly shorter and contained fewer seeds than those of WT plants (Fig. 2c, d). These reproduction-associated phenotypes demonstrated that ureide degradation plays important roles in the late stages of Arabidopsis growth.

\section{Disruption of ureide degradation accelerates leaf senescence in mature plants}

The vegetative-to-reproductive transition generally coincides with the onset of leaf senescence. We therefore examined whether ureide degradation mutants showed accelerated senescence. At 9 WAG, the aln and aah mutants showed more yellowing of the rosette leaves than did WT plants (Fig. 3a \& Additional file 4: Figure S3). To test the early senescence phenotype in these mutants at the molecular level, we analyzed the expression of a canonical senescence marker, SENESCENCE-ASSOCIATED GENE 13 (SAG13), in rosette leaves from 7 -week-old plants by quantitative reverse transcriptionPCR (qRT-PCR) (Fig. 3b). Consistent with their early senescence symptoms, the mutants showed significantly higher levels of SAG13 expression compared with the WT. These findings indicated that defective ureide degradation leads to precocious leaf senescence in mature Arabidopsis plants. 
a

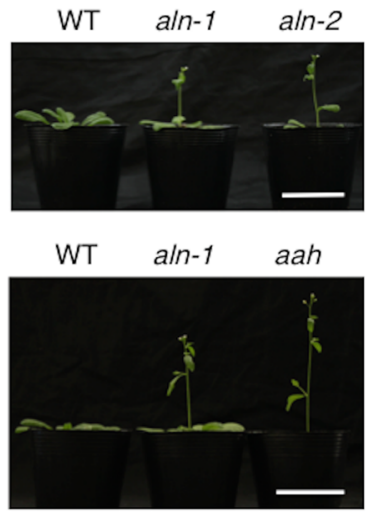

C



b

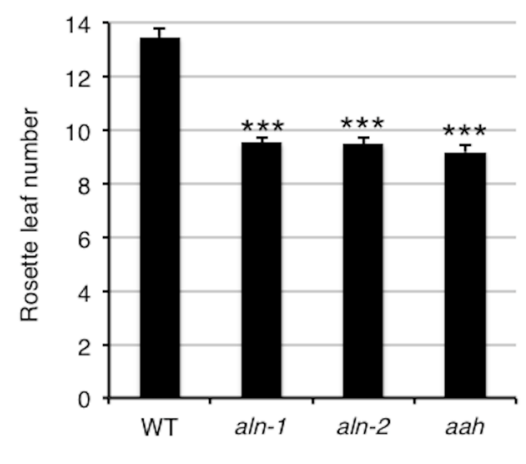

d $\quad$ WT $\quad$ aln-1 $\quad$ aln-2

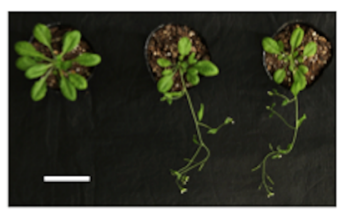

WT aah

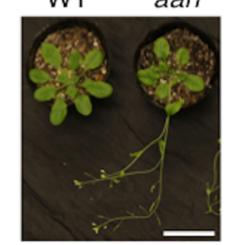

Fig. 1 Early-flowering phenotype of aln and aah mutants. WT, aln-1, aln-2, and aah seeds were sown on soil and grown under long-day conditions. a Growth of representative plants for each genotype at 4 WAG. $\mathbf{b}$ Number of rosette leaves when the primary inflorescence stems were about $1 \mathrm{~cm}$ long $(n \geq 32)$. c Fresh weight (FW) of rosette leaves, petioles, stems, cauline leaves, and siliques from plants at 5 WAG ( $n \geq 9$ ). $\mathbf{d}$ Growth of representative plants for each genotype at 5 WAG. Bar $=5 \mathrm{~cm}$. A linear model with the genotype as a factor was fitted to the data. Asterisks denote significant differences from WT $\left({ }^{*} P<0.05,{ }^{* *} P<0.01\right.$, ${ }^{* *} P<0.001$, two-tailed $t$-tests based on the model)

\section{Disruption of ureide degradation affects $\mathrm{N}$ status but exogenous $\mathrm{N}$ supply did not restore growth of aln mutants}

Since $a l n$ and $a a h$ mutants displayed N-deficiency-like phenotypes as they grew toward maturity (Figs 1, 2, 3), we examined relative $\mathrm{N}$ content, NUE, and carbon-to-nitrogen $(\mathrm{C} / \mathrm{N})$ ratio as indicators of $\mathrm{N}$ status in these mutants. At 9 WAG, all three mutants exhibited significant differences in these parameters relative to WT, with increases in relative $\mathrm{N}$ content but decreases in NUE and $\mathrm{C} / \mathrm{N}$ ratio (Table 1 ). The results suggested that aln and aah mutants were relatively inefficient at using $\mathrm{N}$ for carbon assimilation and dry matter production. On the other hand, the higher relative $\mathrm{N}$ content in ureide-degradation mutants suggested that $\mathrm{N}$ uptake was not affected. To further investigate whether ureides provide substantial $\mathrm{N}$ nutrients, we next tested the effect of $\mathrm{N}$ shortage on plant growth. We hypothesized that the mutants would exhibit growth reduction relative to WT earlier in low-N than $\mathrm{N}$-sufficient conditions, because $\mathrm{N}$ recycling and remobilization processes are activated earlier in low-N conditions to compensate for limited $\mathrm{N}$ supply. Two-week-old aseptically grown seedlings were carefully removed from the medium to avoid damaging the roots, transplanted to a mixture of vermiculite and perlite, and then grown for an additional 4 weeks under $\mathrm{N}$-sufficient $(20 \mathrm{mM})$ or low- $\mathrm{N}(2 \mathrm{mM})$ conditions. It is worth noting that plants grown under these conditions bolted and matured earlier than those under the soil growth conditions described in Fig. 1, 2, 3. At 6WAG, both WT and mutants were apparently at the reproductive stage under these growth conditions, regardless of the $\mathrm{N}$ regime; they had already bolted and developed siliques (Additional file 5: Figure S4). The aah mutants showed DW comparable to WT under $\mathrm{N}$-sufficient conditions, and significantly lower DW than WT under low-N conditions (Fig. 4a). Contrary to our expectation, however, the $a l n-1$ and $a l n-2$ mutants showed significantly decreased DW compared to WT under both $\mathrm{N}$-sufficient and deficient conditions. Irrespective of $\mathrm{N}$ regimes, aln and aah mutants accumulated high levels of allantoin and allantoate, respectively (Fig. 4b), suggesting that $\mathrm{N}$ regimes do not affect ureide biosynthesis. However, WT plants grown under low $\mathrm{N}$ showed significantly decreased allantoate levels, implying that degradation of allantoate is activated by $\mathrm{N}$ deficiency. A small amount 
a
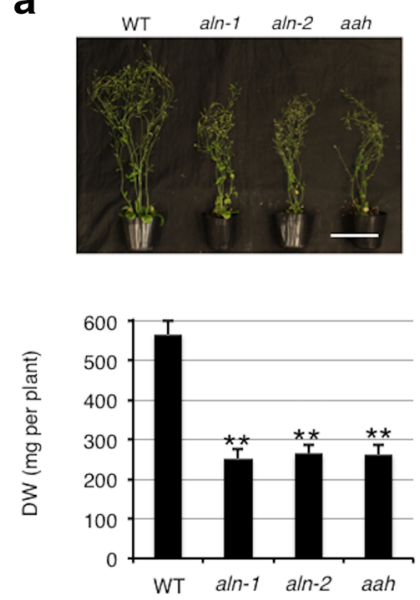

C

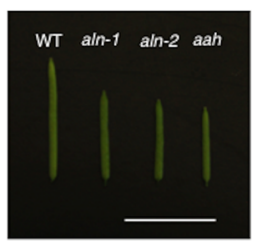

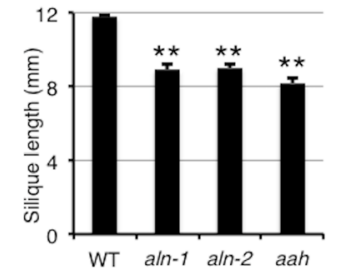

b

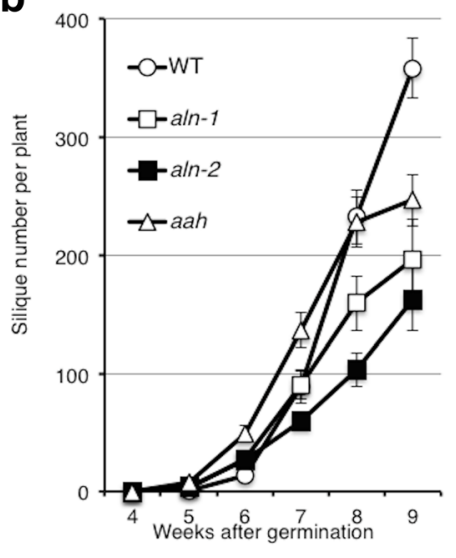

d

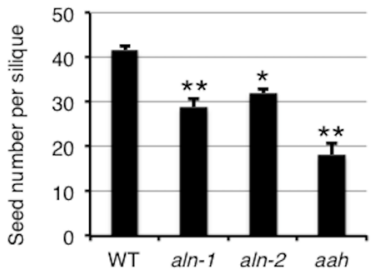

Fig. 2 Reduced growth and fertility of aln and aah mutants. a Growth of representative plants and dry weight (DW) for each genotype at 9 WAG $(n \geq 15)$. Bar $=10 \mathrm{~cm}$. b The number of siliques produced per plant from 4 to 9 WAG $(n \geq 5)$. $\mathbf{c}$ The length of siliques at 9 WAG $(n=10)$. Bar $=1 \mathrm{~cm}$. d The number of seeds in a silique from plants at 9 WAG $(n=6)$. A linear model with the genotype as a factor was fitted to the data. Asterisks denote significant differences from WT $\left({ }^{*} P<0.01,{ }^{* *} P<0.001\right.$, two-tailed $t$-tests based on the model)

of allantoate was detected in the aln-2 plants under $\mathrm{N}$-sufficient conditions, probably due to the spontaneous hydrolysis of allantoin [37].

\section{Disruption of allantoin degradation changed phytohormone balance at 5 WAG}

We previously reported that young seedlings of aln mutants showed significantly enhanced drought and osmotic stress tolerance, along with moderately elevated levels of ABA and JA [27, 28]. Besides controlling stress responses, these hormones affect the levels and activity of other hormones, as well as plant growth and development. Therefore, the impaired growth of the ureide-degradation mutants could be due to altered hormone levels rather than inefficient $\mathrm{N}$ utilization. To test this idea, we determined ABA, JA, jasmonoyl-L-isoleucine (JA-Ile), indole-3-acetic acid (IAA), gibelellin $\left(\mathrm{GA}_{4}\right)$, salicylic acid (SA), trans-zeatin (tZ), $\mathrm{N}^{6}-\left(\Delta^{2}\right.$-isopentenyl)adenine (iP) levels in aboveground parts of soil-grown aln and aah mutants at 5 WAG (Table 2). Although the mutant plants had a higher proportion of reproductive organs than WT at 5 WAG (Fig. 1c, d), we thought that this time point was the best to estimate the effect of stress hormones on the growth reduction, because it was just before the differences in plant sizes between WT and mutants became visible. Compared to the WT, the aln-1 and aln-2 mutants had significantly increased levels of IAA and $\mathrm{GA}_{4}$. In ABA and JA-Ile levels, the aln-2 mutant showed statistically significant differences relative to WT. However, we consider that we do not have significant differences in these hormones in aln plants, since we could not detect them in both alleles. In aah mutants, none of the hormones showed significant changes relative to the WT levels.

\section{Reproductive tissues contain relatively high allantoate levels that are augmented by disruption of AAH}

To investigate the possibility that ureides are utilized as recycled $\mathrm{N}$, we next examined the tissue distribution of allantoin and allantoate in the aboveground parts of Arabidopsis plants, because source-to-sink allocation and redistribution of $\mathrm{N}$ constitute important physiological processes for efficient $\mathrm{N}$ use when plants grow to maturity and produce seeds, or when available $\mathrm{N}$ is limited. At 5 WAG, when WT plants had not bolted or had very small inflorescence stems (Fig. 1c, d), the rosette leaves contained equal levels of allantoin in the leaf blades and petioles and 3-fold higher levels of allantoate in the petioles (Fig. 5a). By this stage, both aln and aah mutants 


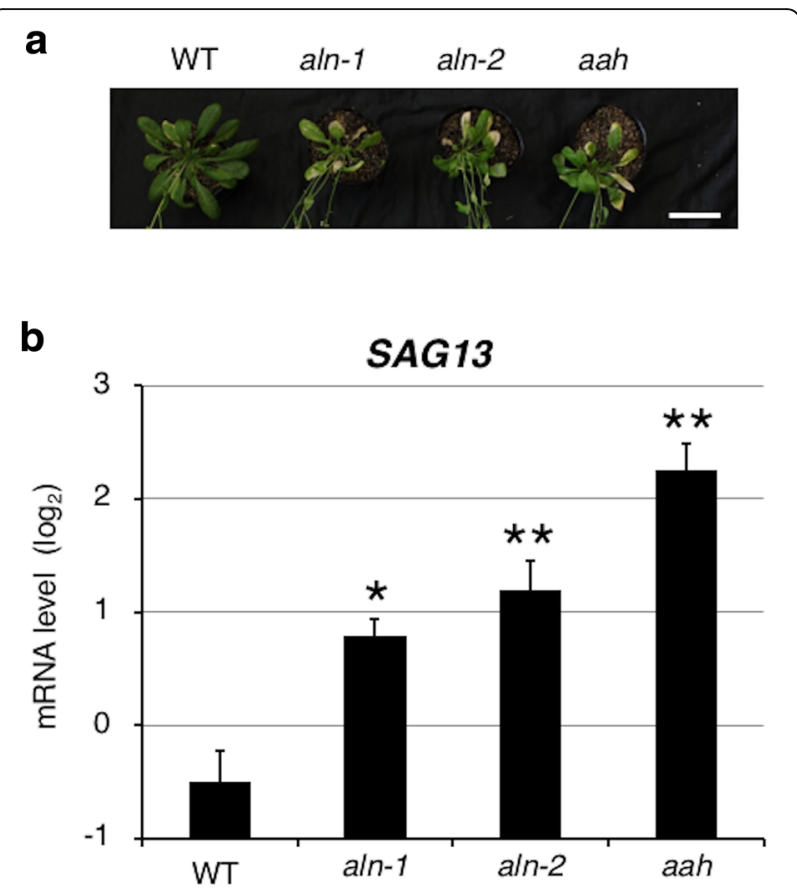

Fig. 3 Accelerated leaf senescence of aln and aah mutants. a Growth of representative plants for each genotype at 9 WAG. Leaves from each genotype are arranged in order of decreasing age from left to right. Bar $=5 \mathrm{~cm}$. b SAG13 expression levels. RNA was extracted from all leaves from plants at 7 WAG. Relative mRNA levels were determined using ACT2, UBC9 and MON1 as reference genes and presented as the $\log _{2}$ of the mean and standard error of three independent biological replicates. A linear model with the genotype as a factor was fitted to the data. Asterisks denote significant differences from WT $\left({ }^{*} P<0.01,{ }^{*} P<0.001\right.$, two-tailed $t$-tests based on the model)

had already started bolting (Fig. 1) and had significantly higher levels of allantoin and allantoate, respectively, than the WT, with allantoate concentrated in inflorescence stems and allantoin distributed more evenly (Fig. 5a). At 9 WAG, WT plants exhibited higher levels of allantoate than allantoin in every tissue examined (leaf blades, petioles, cauline leaves, stems and siliques), and the highest level was detected in siliques (Fig. 5b). Such accumulation profiles were consistent and augmented in the aah mutant, in which allantoate degradation is blocked. Compared to allantoate, allantoin was distributed relatively evenly in the WT and the aah mutant, although the aln mutants appeared to accumulate this ureide slightly more in siliques and cauline leaves than in the other tissues. Possibly, this evenly distributed pattern of allantoin was due to the saturation of this ureide. At both 5 and 9 WAG, a small amount of allantoate was detected from the two alleles of aln mutant plants, probably due to the spontaneous hydrolysis of allantoin [37]. In contrast to other plant parts, mature dry seeds from the WT contained very little allantoate compared with allantoin. Even in the aah mutant seeds, allantoate levels were lower than allantoin (Additional file 6: Figure S5). On the other hand, seed allantoin levels were comparable with, or even higher than those in other plant parts. The seed ureide contents indicate that allantoate in siliques was not localized to seeds, and this ureide does not function as $\mathrm{N}$ storage in Arabidopsis seeds.

\section{Mutations of UPS1 and UPS2 caused growth defects similar to ureide degradation dysfunction but reduced ureide levels in reproductive tissues}

The above results suggested that not only the degradation, but also the tissue partitioning of ureides, which involves membrane transport, is critical for supporting growth at the later stages of Arabidopsis development. Previously, it was shown that impaired $\mathrm{N}$ remobilization due to a block in source-sink transport of amino acids negatively affected seed production [38]. We therefore investigated the effect of disrupting ureide transport on plant growth and the tissue distribution of allantoin and allantoate using ups1 and ups2, two knockout mutants for the Arabidopsis UPS proteins, AtUPS1 and AtUPS2, respectively (Additional file 7: Figure S6). Schmidt et al. [34] previously reported that PTGS of UPS1 and T-DNA insertion of UPS2 did not affect plant growth either on agar plates or soil. However, similar to aln and aah mutants, ups 1 and ups 2 mutants showed early flowering (Fig. 6a), and at 9 WAG under long-day conditions, displayed a reduction in whole-plant DW of $50 \%$ and $25 \%$, respectively, compared with the WT (Fig. 6b). The discrepancy between the present and the previous study might be due to differences in growth conditions and/or observation timing, since detailed methodological information was not available in Schmidt et al. [34].

The tissue distribution of the two ureides was determined at 7 WAG when the WT and the two ups mutants were bolting and developing siliques (Additional file 8:

Table 1 Nitrogen $(\mathrm{N})$ content, nitrogen use efficiency (NUE), and carbon:nitrogen ratio $(\mathrm{C} / \mathrm{N})$ in 9-week-old aln and aah mutants

\begin{tabular}{lllll}
\hline N-status & Genotype & & & \\
\cline { 2 - 5 } Parameter & Wild type & aln-1 & aln-2 & aah \\
\hline N (\% dry weight) & $3.39 \pm 0.13$ & $4.45 \pm 0.13^{* *}$ & $4.10 \pm 0.04^{*}$ & $4.09 \pm 0.15^{*}$ \\
NUE & $29.65 \pm 1.14$ & $22.53 \pm 0.65^{* *}$ & $24.38 \pm 0.26^{* *}$ & $24.55 \pm 0.89^{* *}$ \\
C/N & $11.60 \pm 0.57$ & $8.60 \pm 0.27^{* *}$ & $9.23 \pm 0.22^{* *}$ & $8.89 \pm 0.38^{* *}$ \\
\hline
\end{tabular}

Values are means \pm standard error $(n=4)$

Significant differences using two-tailed $t$-tests based on the linear model at: ${ }^{*}, P<0.01$ and ${ }^{* *}, P<0.001$ 


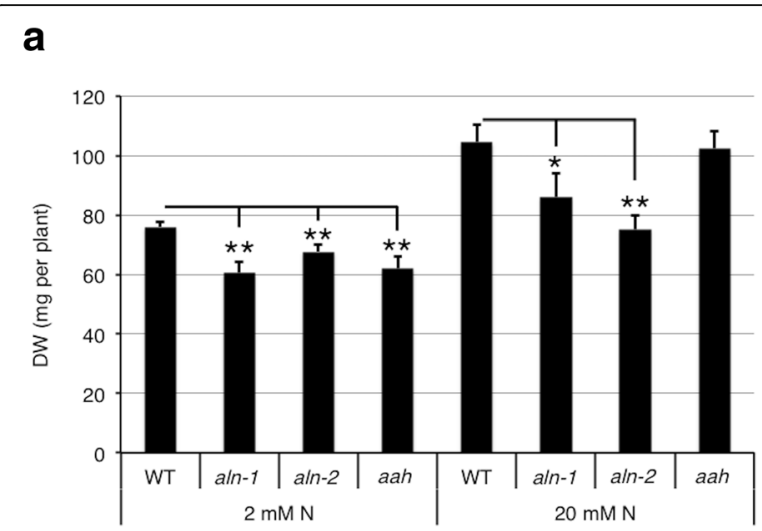

b
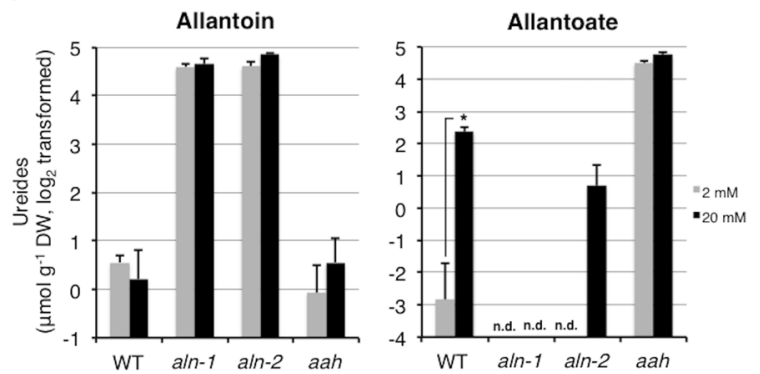

Fig. 4 Effect of nitrogen ( $N$ ) deficiency on the growth of aln and aah mutants. Aseptically grown 2 WAG seedlings were transplanted to pots containing vermiculite and perlite, and then further grown for 4 weeks with weekly irrigation of $\mathrm{N}$-deficient $(2 \mathrm{mM} \mathrm{N})$ and sufficient (20 mM N) nutrient solution. a Dry weight (DW) of whole shoots $(n=16)$. A linear model with the genotype as a factor was fitted to the data. Asterisks denote significant differences between WT and mutant plants ( ${ }^{*} P<0.05,{ }^{* *} P<0.01$, two-tailed $t$-tests based on the model). $\mathbf{b}$ Allantoin and allantoate levels were determined on a dry weight (DW) basis for shoots $(n=3)$. Asterisks denote significant differences in allantoin or allantoate contents between $\mathrm{N}$-deficient and-sufficient conditions for each genotype $\left({ }^{*} P<0.05\right.$, two-tailed $t$-tests). n.d., not detected
Figure S7). AtUPS1 and AtUPS2 have been reported to transport allantoin but exhibit little affinity for allantoate in heterologous assay systems [33, 34]. However, no significant difference was found in allantoin levels between the WT and the mutants in any of the tissues examined (Fig. 6c). To our surprise, compared to the WT, allantoate levels were significantly lower in inflorescence stems of the ups 1 and ups 2 mutants and in siliques of the ups 1 mutant, but increased in rosette leaf blades of the ups 2 mutant. These results showed that, like disruption of ureide degradation, dysfunction of UPS transporters negatively affected the reproductive growth of Arabidopsis. However, the effects on the distribution of the two ureides varied and, notably, allantoate levels decreased in the reproductive tissues of the ups mutants.

\section{Genes involved in ureide synthesis and transport are activated in source tissues at the vegetative-to- reproductive phase transition}

To examine gene expression changes associated with tissue distribution of allantoin and allantoate, we determined changes in transcript levels of genes involved in ureide metabolism (XDH1, $A L N$, and $A A H)$ and transport (AtUPS1 and AtUPS2) in WT plants upon transition from the vegetative (5 WAG, see Fig. 1d) to the reproductive phase (7 WAG; see Additional file 8: Figure S7). qRT-PCR showed that in rosette leaf blades during the growth phase transition, transcript levels for $X D H 1$ and $A L N$ increased, while those for $A A H$ remained constant (Fig. 7a). Consistent with our data, $X D H 1$ and $A L N$ are expressed at relatively high levels in senescent leaves according to publicly available microarray data [39]. In the reproductive organs, the $X D H 1, A L N$ and $A A H$ genes showed higher expression in cauline leaves than in stems; however, stems at 7 WAG showed higher transcript levels of $A L N$ than rosette leaf blades and petioles at 5 WAG. These results suggest that ureide synthesis, particularly of allantoate, is activated in both source (rosette and cauline leaves) and sink (inflorescence stems) organs. Coincident with the increase

Table 2 Phytohormone levels in 5-week-old aln and aah mutants

\begin{tabular}{|c|c|c|c|c|}
\hline \multirow[t]{2}{*}{ Phytohormone } & \multicolumn{4}{|l|}{ Genotype } \\
\hline & $\overline{\text { WT }}$ & aln-1 & aln-2 & $a a h$ \\
\hline$\overline{A B A}\left(n g g^{-1} D W\right)$ & $78.6 \pm 3.2 b$ & $90.8 \pm 6.4 \mathrm{ab}$ & $113.2 \pm 3.3 \mathrm{a}$ & $86.2 \pm 8.1 b$ \\
\hline$J A\left(n g g^{-1} D W\right)$ & $99.5 \pm 60.8 \mathrm{a}$ & $86.0 \pm 26.9 \mathrm{a}$ & $188.0 \pm 71.1 \mathrm{a}$ & $132.5 \pm 57.9 a$ \\
\hline JA-lle (ng g $\left.g^{-1} D W\right)$ & $5.4 \pm 2.3 b$ & $6.6 \pm 1.6 \mathrm{ab}$ & $13.9 \pm 1.0 \mathrm{a}$ & $6.9 \pm 1.6 \mathrm{ab}$ \\
\hline IAA (ng g $\left.g^{-1} D W\right)$ & $180.7 \pm 2.4 \mathrm{C}$ & $402.8 \pm 72.3 b$ & $718.3 \pm 64.5 a$ & $264.5 \pm 40.2 b c$ \\
\hline GA4 (ng g $\left.{ }^{-1} \mathrm{DW}\right)$ & $2.3 \pm 0.5 c$ & $4.6 \pm 0.5 \mathrm{ab}$ & $5.4 \pm 0.2 \mathrm{a}$ & $3.4 \pm 0.2 b c$ \\
\hline $\mathrm{tZ}\left(\mathrm{ng} \mathrm{g}^{-1} \mathrm{DW}\right)$ & $10.8 \pm 0.5 \mathrm{a}$ & $7.6 \pm 1.1 \mathrm{a}$ & $6.4 \pm 1.1 \mathrm{a}$ & $7.8 \pm 1.3 \mathrm{a}$ \\
\hline $\mathrm{iP}\left(\mathrm{ng} \mathrm{g}^{-1} \mathrm{DW}\right)$ & $1.6 \pm 0.1 \mathrm{a}$ & $2.6 \pm 0.4 \mathrm{a}$ & $2.2 \pm 0.3 a$ & $1.7 \pm 0.4 \mathrm{a}$ \\
\hline$S A\left(\mathrm{mg} \mathrm{g}^{-1} \mathrm{DW}\right)$ & $3.2 \pm 0.1 \mathrm{a}$ & $3.9 \pm 1.1 \mathrm{a}$ & $2.9 \pm 0.2 \mathrm{a}$ & $4.0 \pm 0.3 \mathrm{a}$ \\
\hline
\end{tabular}

Values are means \pm standard error $(n=3)$

Different letters indicate significant differences determined by Tukey's HSD test $(P<0.05)$ 


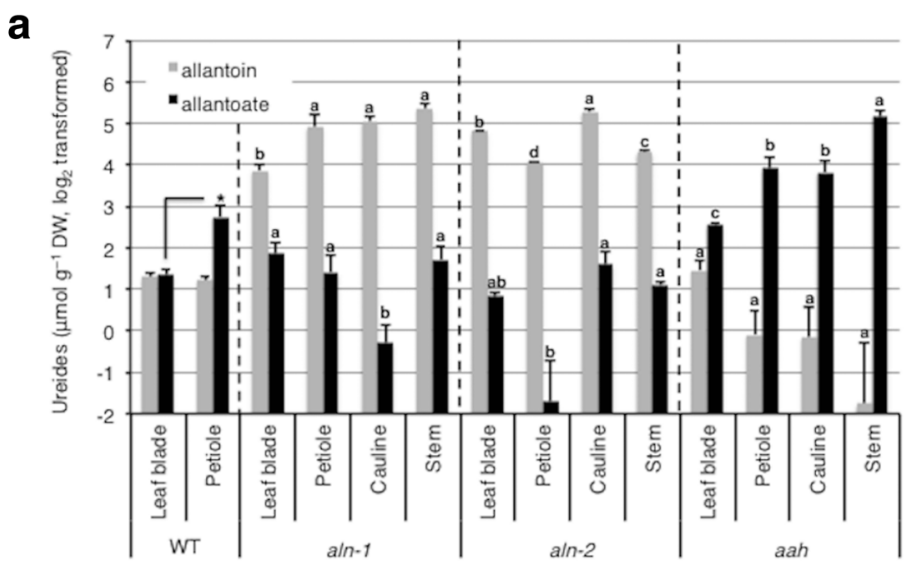

b

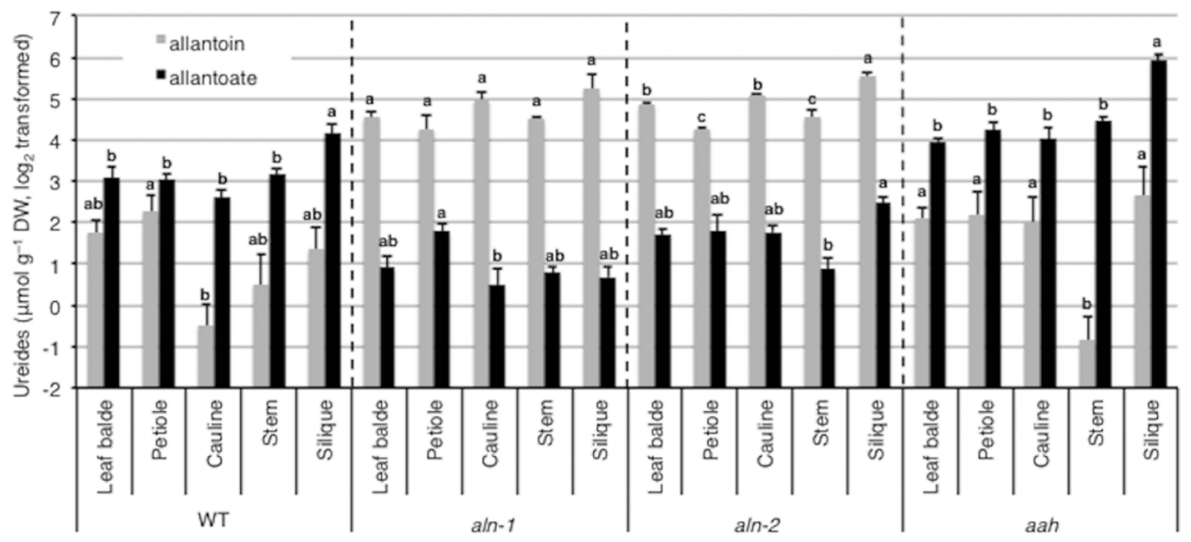

Fig. 5 Ureide concentrations in the aboveground tissue and seeds of aln and aah mutants. a Five WAG plants were cut into leaf blades, petiole, cauline leaves, and inflorescence stems, and allantoin and allantoate levels were determined on a dry weight (DW) basis. Asterisk denotes significant differences of allantoate contents between leaf blade and petiole in 5 -week-old WT $\left(n=3 ;{ }^{*} P<0.05\right.$, two-tailed $t$-test). Different letters indicate significant differences among allantoin or allantoate levels in each mutant line $(n=3 ; P<0.05$, Tukey's HSD test). b Nine WAG plants were cut into leaf blades, petioles, cauline leaves, inflorescence stems, and siliques, and allantoin and allantoate levels were determined on a DW basis. Different letters indicate significant differences among allantoin or allantoate levels in each genotype $(n=3 ; P<0.05$, Tukey's HSD test)

in XDH1 and ALN expression, AtUPS1 and AtUPS2 transcript levels increased in leaf blades and petioles (Fig. $7 \mathrm{~b}$ ). These expression patterns are also consistent with publicly available microarray data; these UPS genes were highly expressed in senescent leaves [39]. In the reproductive organs, AtUPS1 and AtUPS2 genes showed higher expression in cauline leaves than in stems. These results suggest that UPS-mediated ureide partitioning is activated in source organs as Arabidopsis plants transition from vegetative to reproductive growth and the subsequent formation of seeds.

\section{Discussion}

Degradation of the ureides allantoin and allantoate is crucial for utilization of symbiotically fixed $\mathrm{N}$ in ureide-type legumes such as nodulated soybean [40]. In plants other than ureide-type legumes, it has long been regarded as an N-recycling metabolism; however, there is little evidence that ureide degradation is important for growth and development [10]. Seedlings of Arabidopsis mutants with defective ureide degradation (catalyzed by ALN and AAH) indeed grow normally on medium containing sufficient inorganic $\mathrm{N}[18,19,26]$. In this study, we examined the phenotypes of aln-1, aln-2 and aah mutants during and after the transition from vegetative to reproductive growth. When plants were grown long-term under long-day conditions, the three ureide-degradation mutants flowered earlier than the wild type (Fig. 1), and had significant reductions in biomass and seed production (Fig. 2). Moreover, these mutants displayed accelerated leaf senescence (Fig. 3), although allantoin and allantoate have been suggested to function as antioxidant agents [24]. Nourimand and Todd [41] showed that another aln T-DNA insertion line $(a l n-3)$ grown on soil under short-day conditions (8 h light and 16 dark) exhibited comparable growth 
a

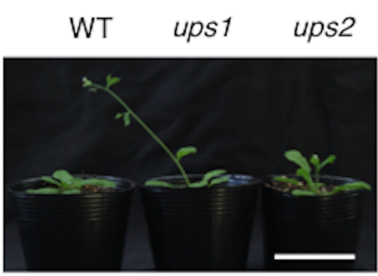

b

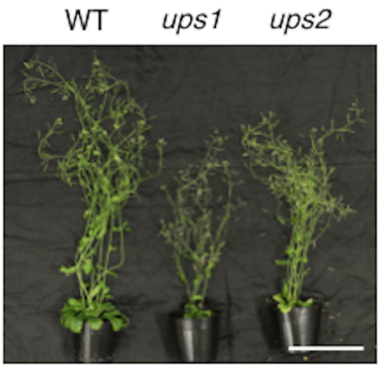

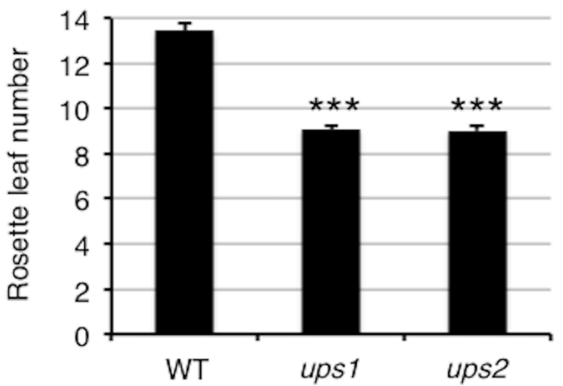

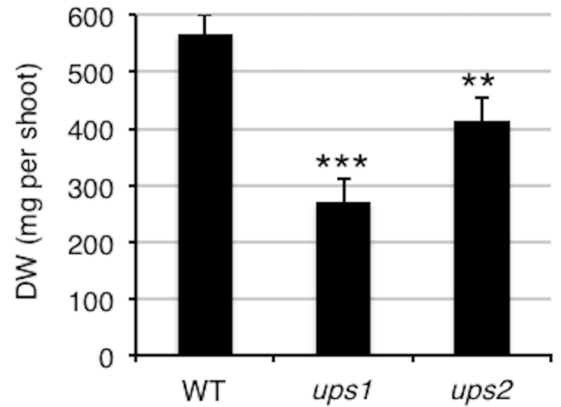

C

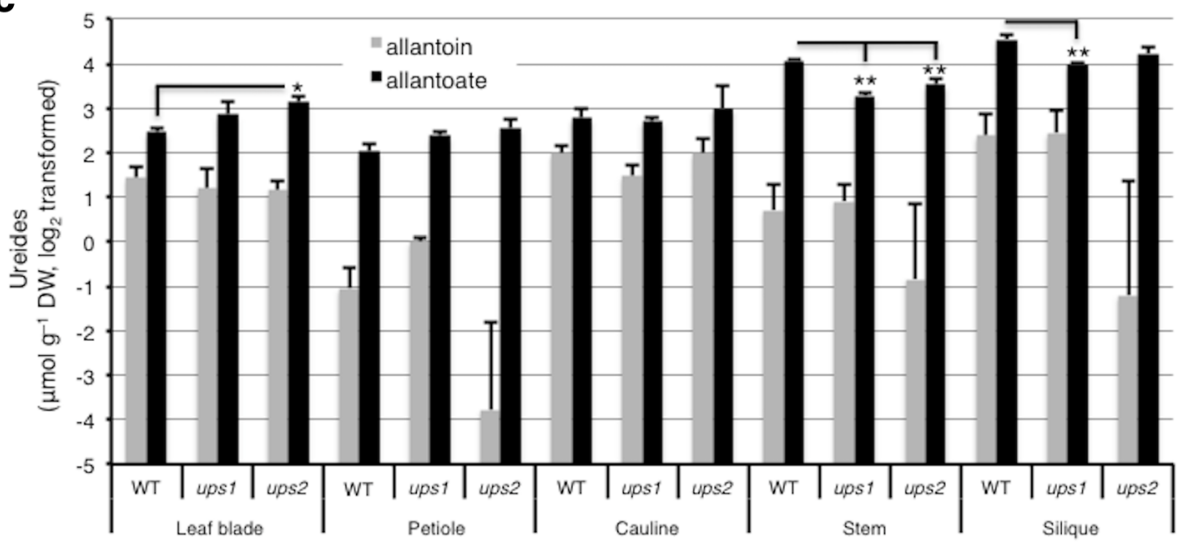

Fig. 6 Growth phenotype and ureide accumulation in the ups 1 and ups 2 mutants. a Early flowering of ups 1 and ups 2 mutants. Representative plants for each genotype are shown at 4 WAG. Bar $=5 \mathrm{~cm}$. Number of rosette leaves when the primary inflorescence stems were about $1 \mathrm{~cm}$ long ( $n \geq 28)$. b Growth of representative plants and dry weight (DW) for each genotype at 9 WAG $(n \geq 12)$. Bar $=10 \mathrm{~cm}$. $\mathbf{c}$ Ureide concentrations in aboveground tissue. Seven WAG plants were cut into leaf blades, petioles, cauline leaves, inflorescence stems, and siliques, and allantoin and allantoate levels were determined on a DW basis $(n=3)$. A linear model with the genotype as a factor was fitted to the data. Asterisks denote significant differences between WT and mutant plants $\left({ }^{*} P<0.05,{ }^{*} P<0.01,{ }^{* *} P<0.001\right.$, two-tailed $t$-tests based on the model)

with WT plants. Because short-day conditions suppress the transition to reproductive stages, the growth phenotypes we observed may be specific for long-day conditions.

\section{Possible reasons for growth reduction: Inefficient $\mathrm{N}$ use and altered stress responses}

At least two possibilities might explain the impaired growth of the ureide-degrading mutants after transition to reproductive growth. The first is that these mutants might experience $\mathrm{N}$ deficiency as they grow to maturity, due to blocked recycling of ureide-derived N. This possibility is supported by the following observations: (i) the mutants displayed symptoms typical of $\mathrm{N}$-starvation (Figs 1, 2, 3); (ii) these symptoms were accompanied by unfavorable $\mathrm{N}$ status, as indicated by decreased NUE and $\mathrm{C}: \mathrm{N}$ ratio (Table 1 ). The possibility that ureides are a source of recycled $\mathrm{N}$ is also supported by the observation that WT plants exposed to low-N conditions had significantly reduced allantoate levels (more than 24-fold lower than in plants grown under N-sufficient conditions; Fig. 4b). It is plausible that this metabolic response reflects the accelerated degradation of allantoate to liberate ammonium for re-assimilation, since Arabidopsis has been shown to utilize allantoin as an $\mathrm{N}$ nutritional source 


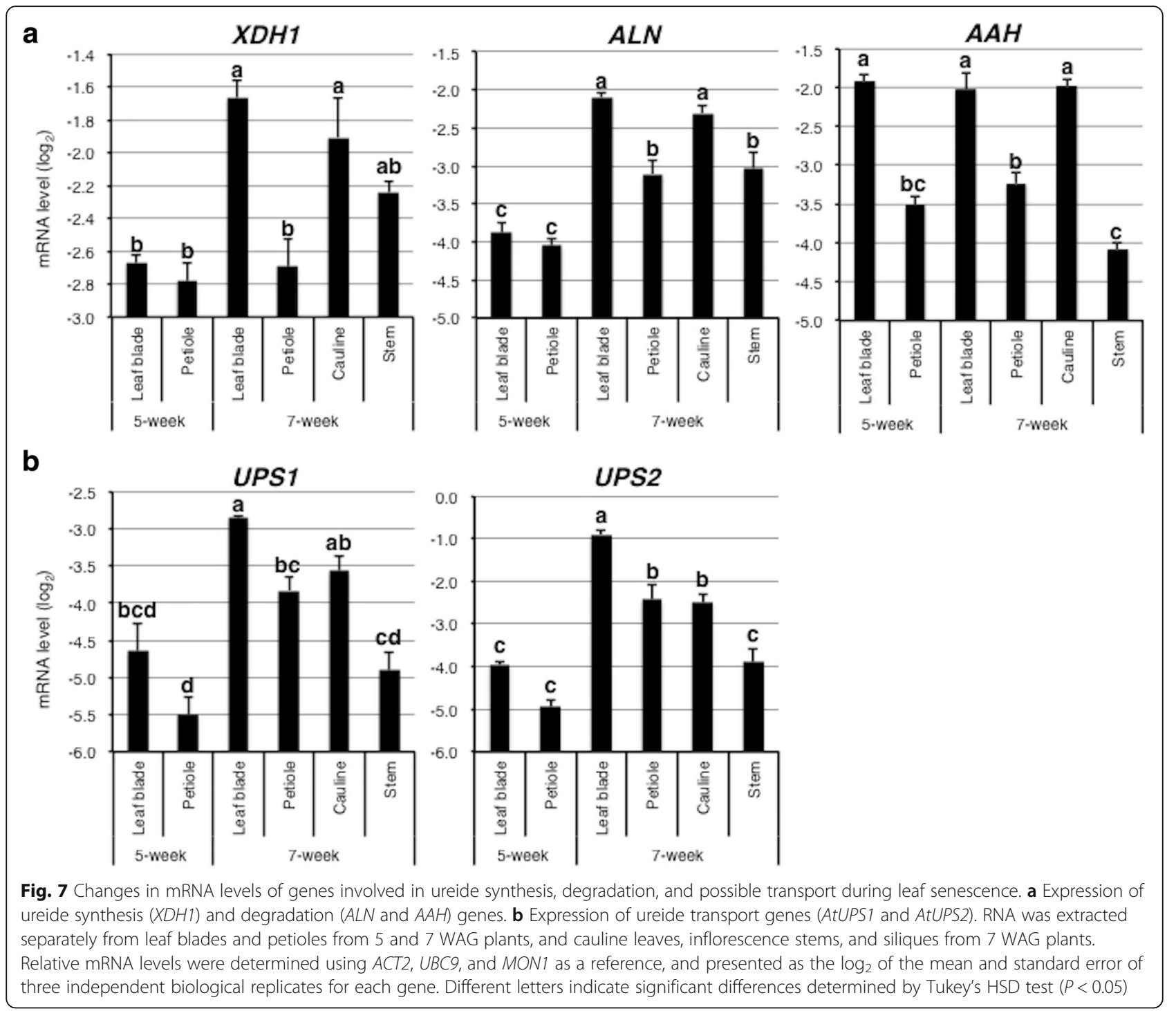

during the early seedling stage $[18,19,33]$. However, growth of aln mutants was not restored to WT levels by sufficient $\mathrm{N}$ supply (Fig. 4a), thus the physiological significance of recycled $\mathrm{N}$ derived from ureides is still questionable and we cannot exclude other possibilities as the cause of growth attenuation.

The second possibility to explain the impaired growth of the ureide-degrading mutants is the antagonistic relationship between plant growth and stress responses [42]. We previously found that allantoin overaccumulation enhances the levels of stress hormones, $\mathrm{ABA}$ and JA, in the aseptically grown 2-week-old seedlings, thereby providing better stress protection at this growth stage [27, 28]. Despite ABA and JA overaccumulation, the aseptically grown aln mutants did not show obvious morphological phenotypes at 2 WAG [27, 28]. In this study, therefore, we tested phytohormone levels at 5 WAG to investigate if $\mathrm{ABA}$ and JA could be factors in growth reduction in ureide-degradation mutants. At 5 WAG, the aln-2 but not aln-1 mutant showed statistically significant differences in ABA and JA-Ile levels (Table 2). It is still difficult to conclude whether ABA and JA affected the growth reduction in the mutants; however, those stress hormones are unlikely to be a main cause of the growth reduction, since neither aln-1 nor aah mutants showed significant differences in ABA and JA levels compared with WT plants (Table 2). It also seems that $\mathrm{ABA}$ and JA had a minor impact on the early onset of flowering, since those hormones are known to suppress the transduction from vegetative to reproductive stages $[43,44]$. Both aln mutants showed significantly increased $\mathrm{GA}_{4}$ and IAA levels. We cannot distinguish if the differences in phytohormone levels are due to the different developmental stages between WT and mutants (Fig. 1c, d). Nevertheless, these hormones might have affected the early transition to the reproductive stage, 
since $\mathrm{GA}_{4}$ promotes flowering and auxin positively regulates GA signaling and biosynthesis [45, 43]. It is also possible that overaccumulation of IAA affected the antagonistic interaction between auxin and cytokinin and restricted shoot growth [46]. However, these altered hormone levels are unlikely to be the sole cause of growth reduction in the ureide-degradation mutants, because the aah mutant did not show significant changes in any phytohormones (Table 2).

There is also a possibility that the phenotypes of ureide-degradation mutants, including altered phytohormone balance, are due to a toxic effect of ureide overaccumulation on the plant cellular functions. Overaccumulation of the ureide precursor uric acid resulted in a severe defect in peroxisome function [15]. While uric acid accumulates in peroxisomes, ureides are localized in the ER. Ureides overacculation might negatively affect ER function in the aln and aah mutants.

\section{Contribution of ureides to $\mathrm{N}$ remobilization and stress protection}

To further explore the importance of ureide utilization in Arabidopsis growth, we also investigated the distribution patterns of ureides. In the aboveground parts, WT plants accumulated substantially more allantoate in influorescence stems and siliques after the onset of reproductive development (Figs 5a, b, 6c). Gene expression data of WT plants are consistent with this ureide accumulation pattern; the upstream genes of allantoin $X D H 1$ and $A L N$ were upregulated during leaf senescence, while $A A H$ remained unchaged (Fig. 7a). In French bean, similarly, allantoate levels were significantly increased in stems after the onset of the reproductive development [47]. This allantoate in French bean was thought to originate from senescent leaves but not nodules, because $\mathrm{N}$-fixation activity was significantly decreased after the onset of reproductive development. Allantoate is the first intermediate substrate to release ammonium in the purine-catabolic pathway, thus it is possible that this acyclic ureide is the preferred form for temporary storage and transport during senescence-induced $\mathrm{N}$ remobilization in both leguminous and non-leguminous plants. In this study, since rosette and cauline leaves of Arabisopsis showed higher $A A H$ expression than petioles and stems (Fig. 7a), it appears that Arabisopsis plants quickly degraded allantoate and subsequently released ammonium in leaves. Presumably, the released ammonium was reassimilated into amino acids and translocated to upper parts of the plants. However, allantoate itself seemed to be translocated from source to sink organs, since this ureide tended to accumulate in repoductive organs of not only WT but also the aah mutant. To our knowledge, the only reported plant proteins that transport allantoate are GmUPS1-1 and GmUPS1-2, two UPS homologs in soybean [30]. Thus, it would be interesting to investigate whether Arabidopsis translocates allantoate via specific transporters. Contrary to other plant parts, seeds contained significantly higher levels of allantoin than allantoate even in aah mutants (Fig. 5c). Allantoin, albeit being one of the two major ureides, responds to a variety of stresses in several species (see [27] and references therein), including Arabidopsis, where it probably plays roles in stress protection as recently reported in the aln mutants [27, 28, 41, 48, 49]. Taking into account its suggested roles in scavenging reactive oxygen species and activating stress responses [24, 27, 28, 50, 51], allantoin might contribute to stress protection during the maturation, dormancy, and germination of seeds, as well as early growth of seedlings. In support of this view, seed allantoin levels in fifteen rice cultivars are highly and positively correlated with seedling survival at low temperatures and under drought conditions [52].

Although they were demonstrated to primarily transport nucleobases such as uracil [34, 35], AtUPS1 and AtUPS2 are still the most likely ureide transporters reported to date in non- $\mathrm{N}_{2}$-fixing species [53]. Two ups mutants decreased allantoate but not allantoin contents in sink tissues (inflorescence stems and siliques). Since allantoin but not allantoate is a substrate of those transporters [33, 34], we think that mutation of UPS genes attenuated allantoin transport from source to sink organs, and allantoin in both source and sink organs was rapidly converted to allantoate. This idea is supported by the gene expression profile of WT plants at 7 WAG (Figs 7). At this stage, compared with 5 WAG, ALN expression levels were relatively higher in both source and sink organs, while UPS1 and UPS2 expression levels were higher only in source but not in sink organs.

We hypothesized that ureide transport duing $\mathrm{N}$ remobilization through AtUPS1 and AtUPS2 is important for efficient $\mathrm{N}$ use and that the ups1 and ups $2 \mathrm{~T}$-DNA insertion mutants would phenocopy, at least partly, the aln and aah mutants as they grew to maturity. The early flowering and reduced growth phenotypes of the two ups mutants supported this prediction (Fig. 6a, b), suggesting that they experienced a perturbation in ureide-derived $\mathrm{N}$ usage. It is unlikely that growth phenotypes of ups mutants during and after onset of reproductive stages are the consequence of attenuated uracil transport, because the importance of uracil salvage declines after germination when de novo synthesis takes over [34].

If ureides are a substantial source of remobilized $\mathrm{N}$, it is interesting to estimate how much $\mathrm{N}$ is recycled and remobilized by ureide degradation. According to data from previous studies, the levels of glutamine and asparagine, two representatives of remobilized N, were roughly 40 and $10 \mu \mathrm{mol} \mathrm{g}^{-1} \mathrm{DW}$ in 6-week-old WT leaves, and 20 and $10 \mu \mathrm{mol} \mathrm{g}^{-1} \mathrm{DW}$ in siliques, respectively, in Arabidopsis $[38,54]$. Since these amido-amino acids contain 
two atoms of $\mathrm{N}$ per molecule, those correspond to approximately 80 and $20 \mu \mathrm{mol} \mathrm{g}{ }^{-1} \mathrm{~N}$ in 6 WAG leaves and 40 and $20 \mu \mathrm{mol} \mathrm{g}^{-1} \mathrm{~N}$ in siliques, respectively, on a dry matter basis. On the other hand, ureide levels in this study were $8.3 \mu \mathrm{mol} \mathrm{g}{ }^{-1} \mathrm{DW}$ (allantoin 2.8 and allantoate $5.5 \mu \mathrm{mol} \mathrm{g}^{-1}$ DW) in 7 WAG WT leaves and

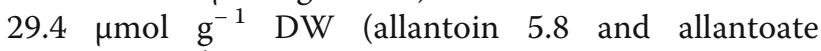
$23.6 \mu \mathrm{mol} \mathrm{g}{ }^{-1} \mathrm{DW}$ ) in siliques (Fig. 6c). Since each ureide has four atoms of $\mathrm{N}$, they correspond to 33.2 and $117.6 \mu \mathrm{mol} \mathrm{g}{ }^{-1} \mathrm{~N}$ in the leaves and siliques, respectively, on a dry matter basis. Given the comparison between amido-amino acids and ureides, it is possible that ureides constitute a substantial percentage of remobilized $\mathrm{N}$ in Arabidopsis.

\section{Conclusions}

Our detailed phenotypic analysis demonstrated that, under long-day conditions, ureide degradation contributes to healthy growth and development during and after transition to the reproductive stage. Mutations blocking ureide degradation affected efficient $\mathrm{N}$ utilization and phytohormone balances, illustrating the physiological importance of this catabolic pathway. Ureides appear to be utilized as remobilized $\mathrm{N}$, since they accumulated more in sink organs such as stems and siliques. Consistent with the phenotypes of ureide-degradation mutants, the impairment of ureide transporters resulted in growth attenuation. Our gene expression analysis using WT plants suggested that ureide degradation and transport are coordinately regulated followed by the progression of senescence.

\section{Materials and methods}

\section{Plant materials and standard growth conditions}

The Arabidopsis thaliana (L.) Heynh. Columbia-0 accession was used as WT. Seeds of the following mutants and T-DNA insertion lines were obtained from the Arabidopsis Biological Resource Center (Ohio State University, Columbus, Ohio, USA): aah (SALK_112631) $[19,27]$, aln-1 (SALK_000325; formerly called $a \ln$ ) [18, 27], aln-2 (SALK_146783) [27], ups1 (SAIL_549_F06; this study), and ups2 (SALK_044551C; also known as ups2-2) [34]. For aseptic growth, seeds were surfacesterilized and cold-treated at $4{ }^{\circ} \mathrm{C}$ for 2 days then placed on half-strength Murashige and Skoog medium containing $1 \%(w / v)$ sucrose and solidified with $0.3 \%$ $(w / v)$ gellan gum. Seeds were germinated and grown at $23{ }^{\circ} \mathrm{C}$ under long-day light conditions (16-h light, 8-h darkness) with a light intensity of $70 \mu \mathrm{mol}$ photons $\mathrm{m}^{-}$ ${ }^{2} \mathrm{~s}^{-1}$. For growth in soil pots, imbibed seeds were sown on garden soil (Hanasaki Monogatari, Akimoto Tensanbutsu, Iga, Japan) which contains the following nutrients: $290 \mathrm{mg} \mathrm{L}^{-1} \mathrm{~N}$; $620 \mathrm{mg} \mathrm{L}^{-1}$ phosphorus; $285 \mathrm{mg} \mathrm{L}^{-1}$ potassium; and small amounts of magnesium, boron, iron, and manganese. Plants were irrigated with tap water as needed to prevent the soil from drying out and supplemented weekly with 1:2000-diluted HYPONeX 6-10-5 fertilizer [neat liquid containing 6\% (w/v) N, 10\% (w/v) phosphoric acid, $5 \%(\mathrm{w} / \mathrm{v})$ water-soluble potassium and micronutrients; HYPONeX Japan Corp., Ltd., Osaka, Japan).

\section{Growth phenotype analysis}

FW was measured for the aerial parts of 5-week-old plants that were separated into rosette leaf blades, petioles, cauline leaves, inflorescence stems, and siliques. For DW measurements, whole shoots of 9 WAG plants were dried in a forced-air oven at $65^{\circ} \mathrm{C}$. Since it was difficult to collect intact roots, they were not used for any analyses. Flowering time was defined as the number of rosette leaves when primary inflorescences were $1 \mathrm{~cm}$ in length. For silique formation, the number of siliques on both the main inflorescence stem and the side branches was counted every week from 4 to 9 WAG. The silique length and seed numbers per silique were evaluated from 10 independent samples where one sample contained 10 representative fully expanded mature siliques selected visually from a single 9-week-old plant. The length of siliques was measured using ImageJ software version 1.45 (http://rsbweb.nih.gov/ij/).

\section{Ureide content}

The ureides allantoin and allantoate were determined by modified methods of Todd and Polacco [18]. Powdered freeze-dried samples were ground in 40 (for WT, ups 1 and ups 2 mutants) or $100 \mathrm{vol}$ (for aln-1, aln-2 and aah mutants) $50 \mathrm{mM}$ potassium phosphate buffer ( $\mathrm{pH} 7.0)$ using a mortar and pestle. Homogenates were transferred to $1.5 \mathrm{~mL}$ microtubes and centrifuged at $18,000 \times g$ for $20 \mathrm{~min}$ at $4{ }^{\circ} \mathrm{C}$. Supernatants were collected in $1.5 \mathrm{~mL}$ microtubes and centrifuged again at $18,000 \times g$ for 5 min at $4{ }^{\circ} \mathrm{C}$ to further spin down residues. Supernatants were collected, and allantoin and allantoate were quantified after chemical transformation to glyoxylate. To transform allantoin to glyoxylate, $10 \mu \mathrm{L}$ of supernatants were mixed with $20 \mu \mathrm{L}$ distilled water and $10 \mu \mathrm{L}$ $0.5 \mathrm{M} \mathrm{NaOH}$, followed by heating at $100{ }^{\circ} \mathrm{C}$ for $8 \mathrm{~min}$. After cooling on ice for $5 \mathrm{~min}$, samples were mixed with $10 \mu \mathrm{L}$ of $0.65 \mathrm{M} \mathrm{HCl}$, heated at $100{ }^{\circ} \mathrm{C}$ for $4 \mathrm{~min}$, and cooled on ice for $5 \mathrm{~min}$. To transform allantoate to glyoxylate, $10 \mu \mathrm{L}$ of supernatants were mixed with $30 \mu \mathrm{L}$ distilled water and $10 \mu \mathrm{L} 0.15 \mathrm{M} \mathrm{HCl}$, and heated at $100{ }^{\circ} \mathrm{C}$ for $4 \mathrm{~min}$. To transform ureidoglycolate to glyoxylate, $10 \mu \mathrm{L}$ of supernatants were mixed with $10 \mu \mathrm{L}$ $0.5 \mathrm{M} \mathrm{NaOH}$, and placed at room temperature for $1 \mathrm{~min}$. After the chemical transformation to glyoxylate, sample volume and $\mathrm{pH}$ were adjusted with $0.4 \mathrm{M} \mathrm{Na}_{2} \mathrm{H}$ $\mathrm{PO}_{4}-\mathrm{KH}_{2} \mathrm{PO}_{4}$ buffer ( $\mathrm{pH}$ 7.0), and $10 \mu \mathrm{L}$ of phenylhydrazine (10 $\mathrm{mg}$ in $3 \mathrm{~mL}$ of water) were added. After $5 \mathrm{~min}$ 
at room temperature, samples were placed on ice, and mixed with $50 \mu \mathrm{l}$ of ice-cold concentrated $\mathrm{HCl}$ and $10 \mu \mathrm{L}$ of potassium ferricyanide $(50 \mathrm{mg}$ in $3 \mathrm{~mL}$ of water). Samples were placed at room temperature at least for $15 \mathrm{~min}$, followed by centrifugation at $15,000 \times g$ for $10 \mathrm{~min}$. Absorbance of supernatants was measured using a spectrophotometer at $535 \mathrm{~nm}$. Allantoin and allantoate concentrations were determined by the subtraction of levels of glyoxylate converted from allantoate and ureidoglycolate, respectively.

\section{Phytohormone quantification}

We used plants at 5 WAG in spite of different developmental stages between WT and mutant plants, since this time seemed just before mutants started showing differences in biomass production. We used whole shoots, but not individual plant parts, since wounding severely affect hormone biosynthesis, especially JA. In addition to wounding effect, stems and cauline leaves in WT plants were too small to collect enough weight for hormone measurements; some WT plants even had not started bolting at 5 WAG. Therefore, in this study, whole aerial parts of 5-week-old plants were freeze-dried and ground to a fine powder. Subsequently, phytohormones were extracted and quantified following the methods of Tsukahara et al. [55] with minor modifications. Samples were mixed with $4 \mathrm{~mL}$ of $80 \%(\mathrm{v} / \mathrm{v})$ acetonitrile containing $1 \%(\mathrm{v} / \mathrm{v})$ acetic acid and known amounts of stable isotope-labeled internal standards at $4{ }^{\circ} \mathrm{C}$ for $1 \mathrm{~h}$. After removal of cell debris by centrifugation at $3000 \times g$ for $10 \mathrm{~min}$, the resultant residues were again extracted with $80 \%(\mathrm{v} / \mathrm{v})$ acetonitrile containing $1 \%(\mathrm{v} / \mathrm{v})$ acetic acid. The two supernatants were combined, evaporated in a vacuum centrifugal evaporator (miVac quattro concentrator, Genevac Ltd., Ipswich, UK) and dissolved in $1 \%(\mathrm{v} / \mathrm{v})$ acetic acid. The extracts were loaded onto a pre-equilibrated Oasis HLB column cartridge (Waters Corporation, Milford, MA, USA). After washing with water acidified with $1 \%$ acetic acid, the column was eluted with $80 \%(\mathrm{v} / \mathrm{v})$ acetonitrile containing $1 \%(\mathrm{v} / \mathrm{v})$ acetic acid. The eluted samples were evaporated to obtain extracts in water acidified with $1 \%$ acetic acid, and loaded onto a pre-equilibrated Oasis MCX column cartridge (Waters). The cartridge was washed with $1 \%$ acetic acid $(\mathrm{v} / \mathrm{v})$, and the acidic fraction was eluted with $80 \%$ acetonitrile containing $1 \%$ acetic acid. A portion of the acidic eluate was used for SA quantification. The cartridge was further washed with $5 \%$ aqueous ammonia, and the basic fraction was eluted with $40 \%$ acetonitrile containing 5\% ammonia and used for $\mathrm{tZ}$ and iP quantification. The remaining acidic fraction was evaporated, dissolved in $1 \%$ acetic acid, and loaded onto pre-equilibrated Oasis WAX column cartridges (Waters). The cartridge was washed with $1 \%$ acetic acid (v/v) and $80 \%$ acetonitrile, and remaining hormones were eluted with $80 \%$ acetonitrile containing $1 \%$ acetic acid. This acidic eluate was used for IAA, GA, ABA, JA, and JA-Ile quantification. All fractions were analyzed on an Agilent 6410 Triple Quadrupole system with a ZORBAX Eclipse XDB-C18 column and MassHunter software version B.01.02 (Agilent Technology, Palo Alto, CA, USA).

\section{Elemental analysis and NUE}

Aerial parts of soil-grown, 9 WAG plants were freeze-dried and ground into fine powder, and $\mathrm{N}$ and $\mathrm{C}$ concentrations were measured by combustion and thermal conductivity separation in a CHN analyzer (Perkin Elmer 2400 Series II CHNS/O Analyzer, Perkin Elmer, Norwalk, Connecticut, USA). NUE was calculated following equation of Steenbjerg and Jakobsen [56].

$$
\mathrm{NUE}=\mathrm{Sw} \div \mathrm{N}_{\text {total }}
$$

where $S w$ is shoot dry weight and $N_{\text {total }}$ is total $\mathrm{N}$ content in the shoot.

\section{$\mathrm{N}$-deficient conditions}

Aseptically grown, 2 WAG seedlings were transplanted into 16-cell trays $(28 \mathrm{~cm} \times 28 \mathrm{~cm} \times 5 \mathrm{~cm}$; each seedling planted in one cell) containing a mixture of vermiculite and perlite $(3: 2[\mathrm{v} / \mathrm{v}])$, and grown for a further 4 weeks, irrigated weekly with culture solution containing either 2 or $20 \mathrm{mM} \mathrm{N}$ (1000 mL per tray; Additional file 9: Table S2). Aerial parts were harvested for DW and ureide measurements.

\section{Gene expression analysis}

qRT-PCR was performed according to the SYBR Green method outlined in Watanabe et al. [27]. Total RNA was extracted using the Nucleospin RNAII extraction kit (Macherey-Nagel GmbH \& Co, Düren, Germany), and cDNA was synthesized from 500 ng of RNA using ReverTra Ace qPCR RT kit (Toyobo, Osaka, Japan). Subsequently, mRNA levels were quantified by qRT-PCR using the KAPA SYBR FAST qPCR Kit (Kapa Biosystems, Inc., Woburn, MA, USA) in a 7300 Real-Time PCR System (Applied Biosystems, Foster City, CA, USA). The PCR program consisted of an initial denaturation at $95^{\circ} \mathrm{C}$ for $10 \mathrm{~min}$, followed by 40 cycles of $95^{\circ} \mathrm{C}$ for $15 \mathrm{~s}$ and $60^{\circ} \mathrm{C}$ for $1 \mathrm{~min}$. The specificity of primers was confirmed using dissociation curve analysis. The relative $\log _{2}$-expression values were obtained by subtracting the $C_{t}$ values for each target gene from the mean of $C_{t}$ value of reference genes, ACTIN 2 (ACT2), UBIQUITIN CONJUGATING ENZYME 9 (UBC9), and MONENSIN SENSITIVITY 1 (MON1). Primer sequences for target genes are listed in Additional file 10: Table S3. 


\section{Statistical analysis}

Quantitative measurements were repeated at least three times and data were expressed as the mean \pm standard error. For the gene expression levels and IAA and ureide contents, $\log _{2}$-transformed values were used to meet requirements for homogeneity of variance. Comparisons of two groups were performed with unpaired two-tailed $t$-tests using Microsoft Excel. Comparison of WT and two or more mutants were performed with two-tailed $t$-tests based on one-factor linear models in the $\mathrm{R}$ programming environment. For balanced data, comparison of three or more groups was performed with one-way ANOVA, followed by Tukey's HSD tests in the R programming environment. For the statistical analysis of gene expression data (Fig. 7), plant age and parts were regarded as one factor because stems and cauline leaves were not included at 5 WAG. For unbalanced data, comparison of three or more groups was performed with Tukey-Kramer multiple comparison tests in the $\mathrm{R}$ programming environment.

\section{Additional files}

\begin{abstract}
Additional file 1: Figure S1. Three-week-old aah mutants grown on gellan gum medium. Two WAG seedlings grown on half-strength Murashige and Skoog medium containing $0.3 \%$ gellan gum were carefully removed to avoid damaging roots, transplanted to new medium and grown for an additional week. (PDF 1951 kb)
\end{abstract}

Additional file 2: Figure S2. Chlorophyll contents of rosette leaves from four WAG plants. Chlorophylls were measured by a hand-held optical sensor (SPAD-502Plus, Konica Minolta Sensing, Inc., Tokyo, Japan) and represented in SPAD units. Values are means \pm standard error $(n \geq 15)$. Different letters indicate significant differences determined by Tukey-Kramer test $(P<0.05)$. (PDF $391 \mathrm{~kb})$

Additional file 3: Table S1. Leaf morphometrics of aln and aah mutants. (DOCX $26 \mathrm{~kb}$ )

Additional file 4: Figure S3. All leaves of representative plants for each genotype at 9 WAG. (PDF $7344 \mathrm{~kb}$ )

Additional file 5: Figure S4. Six WAG plants grown nitrogen-sufficient or -deficient conditions. Aseptically grown 2 WAG seedlings were transplanted to pots containing vermiculite and perlite, and then further grown for 4 weeks with weekly irrigation of N-deficient (2 $\mathrm{mM} \mathrm{N}$ ) or sufficient (20 mM N) nutrient solution. Bar $=5 \mathrm{~cm}$. (PDF $2434 \mathrm{~kb}$ )

Additional file 6: Figure S5. Ureide concentrations in the dry seeds of aln and aah mutants. Asterisks denote significant differences between WT and mutant plants ( $n=3$; ${ }^{* *} P<0.001$, two-tailed $t$-tests). n.d., not detected. (PDF 1069 kb)

Additional file 7: Figure S6. Characterization of ups 1 and ups2 mutants. (a) Diagram of the T-DNA insertion in AtUPS1 in the ups 1 mutant. Arrows indicate PCR primers; white boxes indicate untranslated regions; black boxes indicate exons; black lines indicate introns. (b) PCR-based genotyping of the ups 1 mutant using primers specific to AtUPS1 (F1 and R1) and the left border sequence of the T-DNA (SAIL LB1). (c) Diagram of the TDNA insertion in AtUPS2 in the ups2 mutant. Arrows indicate PCR primers; white boxes indicate untranslated regions; black boxes indicate exons; black lines indicate introns. (d) PCR-based genotyping of the ups 1 mutant using primers specific to AtUPS2 (F2 and R2) and the left border sequence of the T-DNA (LBa1). (e) Semi-quantitative reverse transcription-PCR for estimating AtUPS1 and AtUPS2 mRNA levels in the mutant lines. VHA-A expression was simultaneously analyzed as an internal control. (PDF $865 \mathrm{~kb}$ )
Additional file 8: Figure S7. Growth of representative ups1 and ups2 mutants. Plants were grown at $23{ }^{\circ} \mathrm{C}$ in soil for 7 weeks under long-day conditions. (PDF $257 \mathrm{~kb}$ )

Additional file 9: Table S2. Composition of liquid culture media with low $(2 \mathrm{mM})$ and standard $(20 \mathrm{mM})$ concentrations of inorganic nitrogen. (DOCX 28 kb)

Additional file 10: Table S3. Primers used in this study. (DOCX $32 \mathrm{~kb}$ )

\section{Abbreviations}

AAH: Allantoate amidohydrolase; ABA: Abscisic acid; ACT2: Actin 2; ALN: Allantoinase; C: Carbon; DW: Dry weight; ER: Endoplasmic reticulum; FW: Fresh weight; GA 4 : Gibberellin; IAA: Indole-3-acetic acid; iP: $N^{6}-\left(\Delta^{2}-\right.$ isopentenyl)adenine; JA: Jasmonic acid; JA-lle: Jasmonoyl-L-isoleucine; MON1: Monensin sensitivity 1; N: Nitrogen; NUE: Nitrogen use efficiency; PTGS: Post-transcriptional gene silencing; qRT-PCR: Quantitative reverse transcription-PCR; SA: Salicylic acid; SAG13: Senescence-associated gene 13: tZ: Trans-zeation; UBC9: Ubiquitin conjugating enzyme 9; UPS: Ureide permease; WAG: Weeks after germination; WT: Wild type; XDH: Xanthine dehydrogenase

\section{Acknowledgments}

We thank Fumiaki Katagiri, Jane Glazebrook and Anthony J. Schmitt (University of Minnesota) for critical reading of the manuscript.

\section{Funding}

This work was supported in part by the Ministry of Education, Culture, Sports, Science and Technology, Japan (MEXT) [a Grant-in-Aid for Scientific Research for Innovative Areas (No. 25119717 to A.S.)], the Japan Society for Promotion of Science (JSPS) [a Grant-in-Aid Scientific Research (C) (No. 26440147 to A.S.) and a JSPS Fellowship for Young Scientists (DC, No. 26-5367 to H.T.)], and Tottori University [a Joint Research Program of Arid Land Research Center (to A.S.)]. The hormone analysis was supported by the Japan Advanced Plant Science Network. The funding agencies did not participate in the design of the study, the collection, analysis, and interpretation of data, or in the writing the manuscript.

\section{Availability of data and materials}

The datasets used and/or analysed during the current study are available from the corresponding authors on reasonable request.

\section{Authors' contribution}

HT and AS designed the study. HT performed most of the experiments. SW and ST contributed to the mutant characterization and ureide analysis. HT, TM, ICM, and TH conducted the phytohormone analysis. HT, HS, and AS analyzed the data. HT and AS wrote the manuscript. All authors read and approved the final manuscript.

\section{Ethics approval and consent to participate}

Not applicable.

\section{Consent for publication}

Not applicable.

\section{Competing interests}

The authors declare that they have no competing interests.

\section{Publisher's Note}

Springer Nature remains neutral with regard to jurisdictional claims in published maps and institutional affiliations.

\section{Author details}

${ }^{1}$ Graduate School of Science, Hiroshima University, 1-3-1 Kagamiyama, Higashi-Hiroshima 739-8526, Japan. ${ }^{2}$ Institute of Plant Science and Resources, Okayama University, Kurashiki 710-0046, Japan. ${ }^{3}$ Present Address: Microbial and Plant Genomics Institute, University of Minnesota, Saint Paul, MN 55108, USA. ${ }^{4}$ Present Address: Center for Sustainable Resource Science, RIKEN, Yokohama 230-0045, Japan. 
Received: 3 July 2018 Accepted: 19 October 2018 Published online: 20 November 2018

\section{References}

1. Hörtensteiner S, Feller U. Nitrogen metabolism and remobilization during senescence. J Exp Bot. 2002;53(370):927-37.

2. Masclaux-Daubresse C, Daniel-Vedele F, Dechorgnat J, Chardon F, Gaufichon $L$, Suzuki A. Nitrogen uptake, assimilation and remobilization in plants: challenges for sustainable and productive agriculture. Ann Bot. 2010;105(7): 1141-57.

3. Mae T, Makino A, Ohira K. 1983; changes in the amounts of ribulose bisphosphate carboxylase synthesized and degraded during the life span of rice leaf (Oryza sativa L.). Plant Cell Physiol. 1983;24(6):1079-86.

4. Evans JR, Seemann JR. The allocation of protein nitrogen in the photosynthetic apparatus: costs, consequences, and control. In: Brigs WR, editor. Photosynthesis. New York: Alan R. Liss, Inc; 1989. p. 183-205.

5. Makino A, Sakuma H, Sudo E, Mae T. Differences between maize and rice in $\mathrm{N}$-use efficiency for photosynthesis and protein allocation. Plant Cell Physiol. 2003;44(9):952-6.

6. Buchanan-Wollaston $\mathrm{V}$. The molecular biology of leaf senescence. J Exp Bot. 1997;48(307):181-99.

7. Thomas H, Ougham HJ, Wagstaff C, Stead AD. Defining senescence and death. J Exp Bot. 2003;54(385):1127-32.

8. Stasolla C, Katahira R, Thorpe TA, Ashihara H. Purine and pyrimidine nucleotide metabolism in higher plants. J Plant Physiol. 2003;160(11):1271-95.

9. Zrenner R, Stitt M, Sonnewald U, Boldt R. Pyrimidine and purine biosynthesis and degradation in plants. Ann Rev Plant Biol. 2006;57:805-36.

10. Werner AK, Witte C-P. The biochemistry of nitrogen mobilization: purine ring catabolism. Trends Plant Sci. 2011;16(7):381-7.

11. Werner AK, Medina-Escobar N, Zulawski M, Sparkes IA, Cao F-Q, Witte C-P. The ureide-degrading reactions of purine ring catabolism employ three amidohydrolases and one aminohydrolase in Arabidopsis, soybean, and rice. Plant Physiol. 2013;163(2):672-81.

12. Datta DB, Triplett EW, Newcomb EH. Localization of xanthine dehydrogenase in cowpea root nodules: implications for the interaction between cellular compartments during ureide biogenesis. Proc Natl Acad Sci U S A. 1991:88(11):4700-2.

13. Hesberg C, Hänsch R, Mendel RR, Bittner F. Tandem orientation of duplicated xanthine dehydrogenase genes from Arabidopsis thaliana: differential gene expression and enzyme activities. J Biol Chem. 2004; 279(14):13547-54

14. Yesbergenova Z, Yang G, Oron E, Soffer D, Fluhr R, Sagi M. The plant Mohydroxylases aldehyde oxidase and xanthine dehydrogenase have distinct reactive oxygen species signatures and are induced by drought and abscisic acid. Plant J. 2005;42(6):862-76.

15. Hauck OK, Scharnberg J, Escobar NM, Wanner G, Giavalisco P, Witte C-P. Uric acid accumulation in an Arabidopsis urate oxidase mutant impairs seedling establishment by blocking peroxisome maintenance. Plant Cell. 2014;26(7):3090-100.

16. Lamberto I, Percudani R, Gatti R, Folli C, Petrucco S. Conserved alternative splicing of Arabidopsis transthyretin-like determines protein localization and S-allantoin synthesis in peroxisomes. Plant Cell. 2010;22(5):1564-74.

17. Pessoa J, Sárkány Z, Ferreira-da-Silva F, Martins S, Almeida MR, Li J, Demas AM. Functional characterization of Arabidopsis thaliana transthyretin-like protein. BMC Plant Biol. 2010;10:30.

18. Yang J, Han K-H. Functional characterization of allantoinase genes from Arabidopsis and a nonureide-type legume black locust. Plant Physiol. 2004; 134(3):1039-49.

19. Todd CD, Polacco JC. AtAAH encodes a protein with allantoate amidohydrolase activity from Arabidopsis thaliana. Planta. 2006;223(5):1108-13.

20. Serventi F, Ramazzina I, Lamberto I, Puggioni V, Gatti R, Percudani R. Chemical basis of nitrogen recovery through the ureide pathway: formation and hydrolysis of S-ureidoglycine in plants and bacteria. ACS Chem Biol. 2010;5(2):203-14.

21. Werner AK, Romeis T, Witte C-P. Ureide catabolism in Arabidopsis thaliana and Escherichia coli. Nat Chem Biol. 6(1):19-21.

22. Schubert KR. Products of biological nitrogen fixation in higher plants: synthesis, transport, and metabolism. Ann Rev Plant Physiol. 37:539-74.

23. Nakagawa A, Sakamoto S, Takahashi M, Morikawa H, Sakamoto A. The RNAimediated silencing of xanthine dehydrogenase impairs growth and fertility and accelerates leaf senescence in transgenic Arabidopsis plants. Plant Cell Physiol. 2007;48(10):1484-95.

24. Brychkova G, Alikulov Z, Fluhr R, Sagi M. A critical role for ureides in dark and senescence-induced purine remobilization is unmasked in the Atxdh 1 Arabidopsis mutant. Plant J. 2008;54(3):496-509.

25. Ma X, Wang W, Bittner F, Schmidt N, Berkey R, Zhang L, King H, Zhang $Y$, Feng J, Wen $Y$, et al. Dual and opposing roles of xanthine dehydrogenase in defense-associated reactive oxygen species metabolism in Arabidopsis. Plant Cell. 2016;28(5):1108-26.

26. Werner AK, Sparkes IA, Romeis T, Witte C-P. Identification, biochemical characterization, and subcellular localization of allantoate amidohydrolases from Arabidopsis and soybean. Plant Physiol. 2008;146(2):418-30.

27. Watanabe S, Matsumoto M, Hakomori Y, Takagi H, Shimada H, Sakamoto A. The purine metabolite allantoin enhances abiotic stress tolerance through synergistic activation of abscisic acid metabolism. Plant Cell Environ. 2014; 37(4):1022-36.

28. Takagi H, Ishiga Y, Watanabe $\mathrm{S}$, Konishi T, Egusa M, Akiyoshi N, Matsuura T, Mori IC, Hirayama T, Kaminaka $\mathrm{H}$, et al. Allantoin, a stress-related purine metabolite, can activate jasmonate signaling in a MYC2-regulated and abscisic acid-dependent manner. J Exp Bot. 2016;67(8):2519-32.

29. Pélissier HC, Frerich A, Desimone M, Schumacher K, Tegeder M. PVUPS1, an allantoin transporter in nodulated roots of French bean. Plant Physiol. 2004; 134(2):664-75.

30. Collier R, Tegeder M. Soybean ureide transporters play a critical role in nodule development, function and nitrogen export. Plant J. 2012;72(3):355-67.

31. Carter AM, Tegeder M. Increasing nitrogen fixation and seed development in soybean requires complex adjustments of nodule nitrogen metabolism and partitioning processes. Curr Biol. 2016;26(15):2044-51.

32. Pélissier HC, Tegeder M. PVUPS1 plays a role in source-sink transport of allantoin in French bean (Phaseolus vulgaris). Funct Plant Biol. 2007;34(4): 282-91

33. Desimone $M$, Catoni $E$, Ludewig U, Hilpert M, Schneider A, Kunze R, Tegeder M, Frommer WB, Schumacher K. A novel superfamily of transporters for allantoin and other oxo derivatives of nitrogen heterocyclic compounds in Arabidopsis. Plant Cell. 2002;14(4):847-56.

34. Schmidt A, Su Y-H, Kunze R, Warner S, Hewitt M, Slocum RD, Ludewig U, Frommer WB, Desimone M. UPS1 and UPS2 from Arabidopsis mediate high affinity transport of uracil and 5-fluorouracil. J Biol Chem. 2004;279(43): 44817-24.

35. Schmidt A, Baumann N, Schwarzkopf A, Frommer WB, Desimone M. Comparative studies on Ureide permeases in Arabidopsis thaliana and analysis of two alternative splice variants of AtUPS5. Planta. 2006;224(6): 1329-40.

36. Forstheofel NR, Wu Y, Schulz B, Bennett MJ, Feldmann KA. T-DNA insertion mutagenesis in Arabidopsis: prospects and perspective. Aust J Plant Physiol. 1992;19(4):353-66.

37. Vogels GD, de Windt FE, Bassie. Hydeolysis and racemization of allantoin. Recl Trav Chim 1969;88(8):940-950.

38. Santiago JP, Tegeder M. Connecting source with sink: the role of Arabidopsis AAP8 in phloem loading of amino acids. Plant Physiol. 2016; 171(1):508-21.

39. Winter D, Vinegar B, Nahal H, Ammar R, Wilson GV, Provart NJ. An "electronic fluorescent pictograph" browser for exploring and analyzing large-scale biological data sets. PLoS One. 2007;2(8):e718.

40. Smith PMC, Atkins CA. Purine biosynthesis. Big in cell division, even bigger in nitrogen assimilation. Plant Physiol. 2002;128(3):793-802.

41. Nourimand $M$, Todd CD. Allantoin increases cadmium tolerance in Arabidopsis via activation of antioxidant mechanisms. Plant Cell Physiol. 2016;57(12):2485-96

42. Kim J-S, Mizoi J, Kidokoro S, Maruyama K, Nakashima K, Mitsuda N, Takiguchi Y, Ohme-Takagi M, Kondou Y, Yoshizumi T, et al. Arabidopsis growthregulating factor7 functions as a transcriptional repressor of abscisic acidand osmotic stress-responsive genes, including DREB2A. Plant Cell. 2012; 24(8):3393-405.

43. Weiss $D$, Ori N. Mechanisms of cross talk between gibeberellin and other hormones. Plant Physiol. 2007;144(3):1240-06.

44. Huang H, Liu B, Liu L, Song S. Jasmonate action in plant growth and development. J Exp Bot. 2017;68(6):1349-59.

45. Wilson RN, Heckman JW, Somerville CR. Gibberellin is required for flowering in Arabidopsis thaliana under short days. Plant Physiol. 1992; 100(1):403-8. 
46. Su Y-H, Liu Y-B, Zhang X-S. Auxin-cytokinin interaction regulates meristem development. Mol Plant. 2011;4(4):616-25.

47. Díaz-Leal JL, Gálvez-Valdivieso G, Fernández J, Pineda M, Alamillo JM. Developmental effects on ureide levels are mediated by tissue-specific regulation of allantoinase in Phaseolus vulgaris L. J Exp Bot. 2012;63(11): 4095-106.

48. Irani S, Todd CD. Ureide metabolism under abiotic stress in Arabidopsis thaliana. J Plant Physiol. 2016;199:87-95.

49. Lescano Cl, Martini C, Gonzáles CA, Desimone M. Allantoin accumulation mediated by allantoinase downregulation and transport by Ureide permease 5 confers salt stress tolerance to Arabidopsis plants. Plant Mol Biol. 2016;91(4-5):581-95

50. Watanabe S, Nakagawa A, Izumi S, Shimada H, Sakamoto A. RNA interference-mediated suppression of xanthine dehydrogenase reveals the role of purine metabolism in drought tolerance in Arabidopsis. FEBS Lett. 2010;584(6):1181-6.

51. Watanabe S, Kounosu Y, Shimada H, Sakamoto A. Arabidopsis xanthine dehydrogenase mutants defective in purine degradation show a compromised protective response to drought and oxidative stress. Plant Biotechnol. 2014;31(2):173-8

52. Wang $\mathrm{P}$, Kong $\mathrm{C}-\mathrm{H}$, Sun $\mathrm{B}, \mathrm{Xu} \mathrm{X}-\mathrm{H}$. Distribution and function of allantoin (5ureidohydantoin) in rice grains. J Agric Food Chemistry. 2012;60(11):2793-8.

53. Girke C, Daumann M, Niopek-Witz S, Möehlmann T. Nucleobase and nucleoside transport and integration into plant metabolism. Front Plant Sci. 2014;5:443.

54. Ladwig F, Stahl M, Ludewig U, Hirner AA, Hammes UZ, Stadler R, Harter K, Koch W. Siliques are Red1 from Arabidopsis acts as a bidirectional amino acid transporter that is crucial for the amino acid homeostasis of siliques. Plant Physiol. 2012;158(4):1643-55.

55. Tsukahara K, Sawada H, Kohno Y, Matsuura T, Mori IC, Terao T, loki M, Tamaoki M. Ozone-induced rice grain yield loss is triggered via a change in panicle morphology that is controlled by ABERRANT PANICLE ORGANIZATION 1 gene. PLoS One. 2015:10(4):e0123308.

56. Steenbjerg F, Jakobsen ST. Plant nutrition and yield curves. Soil Sci. 1963; 95(1):69-88

Ready to submit your research? Choose BMC and benefit from:

- fast, convenient online submission

- thorough peer review by experienced researchers in your field

- rapid publication on acceptance

- support for research data, including large and complex data types

- gold Open Access which fosters wider collaboration and increased citations

- maximum visibility for your research: over $100 \mathrm{M}$ website views per year

At $\mathrm{BMC}$, research is always in progress.

Learn more biomedcentral.com/submissions 